\title{
Video Article \\ Three-dimensional Imaging of Bacterial Cells for Accurate Cellular Representations and Precise Protein Localization
}

\author{
Benjamin P. Bratton ${ }^{2}$, Brody Barton ${ }^{1}$, Randy M. Morgenstein ${ }^{1}$ \\ ${ }^{1}$ Department of Microbiology and Molecular Genetics, Oklahoma State University \\ ${ }^{2}$ Department of Molecular Biology and Lewis-Sigler Institute of Integrative Genomics, Princeton University
}

Correspondence to: Randy M. Morgenstein at randy.morgenstein@okstate.edu

URL: https://www.jove.com/video/60350

DOI: doi: $10.3791 / 60350$

Keywords: Immunology and Infection, Issue 152, protein localization, cell shape, microscopy, three-dimensional microscopy, morphogenesis, forward convolution, computational image processing

Date Published: 10/29/2019

Citation: Bratton, B.P., Barton, B., Morgenstein, R.M. Three-dimensional Imaging of Bacterial Cells for Accurate Cellular Representations and Precise Protein Localization. J. Vis. Exp. (152), e60350, doi:10.3791/60350 (2019).

\section{Abstract}

The shape of a bacterium is important for its physiology. Many aspects of cell physiology such as cell motility, predation, and biofilm production can be affected by cell shape. Bacterial cells are three-dimensional (3D) objects, although they are rarely treated as such. Most microscopy techniques result in two-dimensional (2D) images leading to the loss of data pertaining to the actual 3D cell shape and localization of proteins Certain shape parameters, such as Gaussian curvature (the product of the two principal curvatures), can only be measured in 3D because $2 \mathrm{D}$ images do not measure both principal curvatures. Additionally, not all cells lie flat when mounting and 2D imaging of curved cells may not accurately represent the shapes of these cells. Accurately measuring protein localization in 3D can help determine the spatial regulation and function of proteins. A forward convolution technique has been developed that uses the blurring function of the microscope to reconstruct $3 \mathrm{D}$ cell shapes and to accurately localize proteins. Here, a protocol for preparing and mounting samples for live cell imaging of bacteria in $3 \mathrm{D}$ both to reconstruct an accurate cell shape and to localize proteins is described. The method is based on simple sample preparation, fluorescent image acquisition, and MATLAB-based image processing. Many high-quality fluorescent microscopes can be simply modified to take these measurements. These cell reconstructions are computationally intensive and access to high-throughput computational resources is recommended, although not necessary. This method has been successfully applied to multiple bacterial species and mutants, fluorescent imaging modalities, and microscope manufacturers.

\section{Video Link}

The video component of this article can be found at https://www.jove.com/video/60350/

\section{Introduction}

Cells of all types regulate their shapes for specific functions. For example, neurons are shaped differently than blood cells and have different functions. Similarly, bacterial cells come in a variety of shapes and sizes, although the purpose of these shapes is not always known ${ }^{1,2}$. Therefore, it is important that the shape of bacterial cells be accurately determined. The method outlined shows an easily implemented way to collect data suitable for the 3D analysis of most live or fixed bacterial cells.

The method described enables one to take 3D images of bacterial cells in order to accurately represent the 3D cell shape of the sample and to precisely localize proteins within these shapes. Traditional microscopy techniques take 2D images, which is problematic when studying cells that have abnormal or nonsymmetrical shapes, such as mutants of Escherichia coli, or curved bacteria such as Vibrio cholerae and Helicobacter pylori. While high-resolution 3D images are the key input to this method, the method does not return a resolution-enhanced image. Rather, this method reconstructs the 3D surface coordinates and shape of the cell using a forward convolution algorithm using active contours and the apparent blurring function of the microscope ${ }^{3}$ (Figure 1). It has been used to study the bacterial actin homolog MreB in $E$. coll ${ }^{4,5,6,7}$, the novel periskeletal element CrvA in V. cholerae ${ }^{8}$, and the putative bactofilin CcmA in Helicobacter pylori ${ }^{9}$ (Figure 2).

The localization of proteins can give insight into their functions. For example, proteins involved in cell division are normally localized to the midcell ${ }^{10,11}$. High-throughput studies have been undertaken to localize all the proteins of a bacterium in hopes of gaining insight into their functions ${ }^{12}$. Unfortunately, these studies were performed with 2D imaging and 1D or 2D analysis, making it impossible to measure specific aspects of protein localization, such as localization to cellular geometric features.

For example, MreB, a dynamic protein required for the rod shape of many bacteria, is hypothesized to work by directing the localization of cell wall synthesis, and its localization mirrors the localization of cell wall synthesis ${ }^{7,13}$. MreB from multiple species shows geometric enrichment ${ }^{4,6,7,14,15}$. Dynamic surface polymers, such as MreB, may couple the geometry of the surface to the time averaged enrichment profile ${ }^{16}$ and may be able to orient to specific geometries by minimizing the energy associated with binding to the membrane ${ }^{17}$. While the importance of twisting, bundling, bending, and dynamics has not been entirely resolved for MreB, it is important to note that accurate measurements of both principal curvatures of a surface require a full $3 \mathrm{D}$ representation of the cell. Therefore, to most accurately measure the curvatures to 
which proteins localize, it is preferential to use $3 \mathrm{D}$, rather than $2 \mathrm{D}$ imaging. $3 \mathrm{D}$ imaging eliminates the need to computationally estimate those curvatures which cannot be measured in $2 \mathrm{D}$, an estimate that might not be accurate in asymmetrical cells ${ }^{18}$.

Although 2D imaging of cells is quicker and does not require as much postimaging computational work, 3D imaging provides a more accurate representation of the cell, as well as the ability to measure surface features, such as curvature, which cannot be measured in 2D. Therefore, as 3D imaging becomes more commonplace, new insights into cell shape and protein localization will become possible.

Protocol

\section{Sample Preparation}

1. Make E. coli fluorescent for imaging either by engineering them to express a cytoplasmic fluorescent protein ${ }^{6}$ or using a membrane dye ${ }^{5}$. Other bacterial species can be used instead of $E$. coli.

1. Transform cells via electroporation with a plasmid that encodes the cytoplasmic mCherry fluorescent protein. NOTE: Different fluorescent proteins of other colors of can be used.

2. Grow the transformants on an LB plate with the appropriate antibiotic at $37^{\circ} \mathrm{C}$. Obtain single colonies and identify positive clones by microscopy. Antibiotic resistant colonies that appear red under the microscope contain the plasmid carrying mCherry.

2. Grow $2 \mathrm{~mL}$ of the overnight culture in LB liquid media with the appropriate antibiotics at $37^{\circ} \mathrm{C}$ in a shaking incubator. Use either a colony from a plate or a small amount of a freezer stock as the inoculum.

NOTE: Use the media necessary for the bacterial cells selected for the experiment.

3. Subculture the overnight culture $1: 1,000$ into $5 \mathrm{~mL}$ of fresh LB media and let the cells grow to exponential phase at $37^{\circ} \mathrm{C}$ in a shaking incubator for 3-4 h. Measure the $\mathrm{OD}_{600}$ of the cells to confirm they are in the exponential phase (i.e., $\mathrm{OD}_{600}=0.2-0.4$ ).

NOTE: Imaging can be performed at any growth stage depending on the experiment being performed.

\section{Slide Preparation}

NOTE: It is important to use media that has low autofluorescence. Any medium which has low autofluorescence can be used. In this experiment, $1 \%$ agarose M63 minimal media pads and sealing with $\mathrm{VaLaP}^{19}$ is required to image cells in 3D.

1. Prepare a $1 \%$ solution of agarose in $20 \mathrm{~mL}$ of minimal media.

1. Microwave the solution until the agarose is fully dissolved and the solution appears clear.

2. Keep the solution in a $60{ }^{\circ} \mathrm{C}$ water bath until ready to use.

NOTE: This solution can be solidified and melted as needed or can be aliquoted into smaller amounts that are melted as needed. After multiple uses the media may become discolored and should be replaced.

2. Melt VaLaP sealant on a hot plate at $80-100{ }^{\circ} \mathrm{C}$

NOTE: Prepare VaLaP, to be used as a sealant, by melting $50 \mathrm{~g}$ each of petroleum jelly, lanolin, and paraffin together in a beaker on a hot plate at $80-100{ }^{\circ} \mathrm{C}$. This large amount of VaLaP can be stored at room temperature indefinitely and will be enough for $>500$ slides. Heating at $>100{ }^{\circ} \mathrm{C}$ will cause it to degrade and its color will darken.

3. Place two stacks each of three $20 \mathrm{~mm} \times 20 \mathrm{~mm}$ cover slips on the opposite ends of a slide (six total coverslips).

4. Pipette $200 \mu \mathrm{L}$ of agarose pad solution onto the slide.

NOTE: If not using an engineered fluorescent stain, a membrane dye may be added to the agarose pad solution before this step. Resuspend the dye in water and add dye to $1 \mathrm{~mL}$ of the agarose pad solution to a final concentration of $5 \mu \mathrm{g} / \mathrm{mL}$.

5. Immediately and firmly place a second slide down on the stack of cover slips to flatten the agar and let it solidify for 1 min at room temperature.

6. Carefully slide off the top slide.

7. Use the large end of a $200 \mu \mathrm{L}$ pipette tip to cut out individual pads from the gel ( $5 \mathrm{~mm}$ in diameter) on the slide and discard malformed or unneeded gel.

NOTE: If imaging multiple strains or conditions, a separate pad will be needed for each one. If only one strain is to be imaged, make 3-4 pads to help support the coverslip.

8. Pipette cells up and down multiple times to disrupt cell clumps and ensure the culture is well mixed. Pipette $1 \mu \mathrm{L}$ of subculture from step 1.3 onto a pad.

NOTE: Cell shape reconstructions require individual cells that are not touching other cells. If using stationary phase or high cell density cultures, it may be necessary to dilute the sample 1:10 before placing it on the pad.

9. Let the sample air dry for 5-10 min. Ensure that the droplet is completely absorbed into the pad. If any liquid remains, the cells will move around in the liquid and cannot be imaged.

10. Place a cover slip on the top of the pads.

11. Seal the cover slip with melted VaLaP by gently brushing around the edge of the cover slip with a cotton swab. Make sure to keep it away from the top of the cover slip where the objective will touch. The VaLaP will harden in a matter of seconds, sealing the sample.

12. Immediately image the sample.

NOTE: The sample should be imaged as soon as the slide is prepared. The cells can grow on the pads, and if they divide the reconstructions will be more difficult.

\section{ImagingRequirements}

1. Ensure that the $z$ or focusing axis of the microscope can make precise movements of less than $50 \mathrm{~nm}$. Use $z$ piezo stages (see Table of Materials), available on research grade microscopes, because typical motorized focus devices are unable to provide this precision. 
NOTE: Assuming the Nyquist-Shannon sampling criterion ${ }^{20}$, one needs to have the ability to move $0.5 x$ the smallest desired step size, or $2 x$ the desired spatial frequency. For the $100 \mathrm{~nm}$ steps needed in this protocol, a stage with a precision of $50 \mathrm{~nm}$ or less is required.

2. Ensure that the microscope contains a 100x objective with a minimum numerical aperture (NA) of 1.45 .

1. Collect z-stacks of between 200-400 different cells to ensure enough cells are obtained for downstream applications.

NOTE: The number of cells needed depends on the underlying variability of the sample of interest. Some of the cells collected at this point will not make it through the reconstruction steps.

3. Ensure that the $z$-stack matches the settings used for the shape channel if a secondary fluorescent channel (protein, metabolic label, etc.) is measured.

\section{Imaging}

1. Insert the sealed slide onto the microscope and allow it sit for $5 \mathrm{~min}$ to equilibrate the temperature with the surroundings, because the microscope room may be at a different temperature than the sample preparation room.

2. Take a fluorescent z-stack of the sample.

NOTE: The z-stack should entirely cover the sample with a z-spacing less than the depth of field. For a $1.45 \mathrm{NA} 100 \mathrm{x}$ objective and $\sim 1 \mu \mathrm{m}$ thick E. coli cells, 40 steps at $100 \mathrm{~nm}$ per step work well. For larger cells or cells that do not lie perfectly flat on the surface, 50 or more steps may be necessary. Include enough steps and ensure that the sample is fully blurred above and below.

1. Use the software associated with the microscope (see Table of Materials) to control the microscope.

2. Focus on the middle of the cell using the microscope focus wheels. Under ND acquisition check the $\mathbf{Z}$ box to take a $\mathbf{Z}$-stack. Click the Home button to set the middle of the cell as the starting point. Set the Step size to $0.1 \mu \mathrm{m}$ and set the Range to $4 \mu \mathrm{m}$. Make sure that the $Z$ device is set to the piezo stage.

3. Set the fluorescent channels under the Lambda Window to the settings for the fluorescent molecules being imaged. In this experiment GFP and mCherry were used.

NOTE: Take an additional z-stack with the same Step Size and Range in the second color channel if the 3D distribution of an additional fluorescent channel is desired. In this experiment, cytoplasmic mCherry was used to determine cell shape and MreB-GFP was used as a second color channel ${ }^{21}$.

4. Ensure that the Order of Experiment is set to lambda ( $z$ series) so that it will take a complete z-stack in each color channel before switching.

5. Click Run Now to start the image acquisition and save the file onece one or both z-stacks are complete.

6. Move to a new area on the pad and repeat steps 4.2.2-4.2.5.

\section{Cell Reconstruction}

1. Crop individual cells and save the images as a stacked tiff file so that there is only one cell per file. Ensure that this cell is well isolated from any other cells (i.e., roughly $5 x$ the full-width half-maximum of the blurring function in $x y$.

NOTE: This can be done using freely available image analysis software (see Table of Materials).

1. Draw a box around an individual cell and Duplicate that cell $2 x$, once for each channel. Make sure the duplicate hyperstack box is checked and change the channel to either 1 or 2 , making sure that the slices include the entire $z$-stack.

NOTE: If imaging only the shape of the cells and not an additional fluorescent protein, only one channel will be present.

2. Once both stacks are available go to Images | Stacks | Tools | Concatenate to combine the images with the protein channel first and the shape channel second.

3. Save the new image as a tiff file.

2. Measure the blurring function of the microscope using subdiffraction limited fluorescent beads ${ }^{22}$. This needs to be done for each microscope and microscope objective but can be performed before or after imaging the samples of interest.

1. Average together multiple independent beads with some manual intervention using available software (see Table of Materials). NOTE: The final product should be a 3D image of the blurring function with the same xyz spacing as the samples of interest.

3. Run the forward convolution cell shape reconstruction scripts using available software. The latest version of these scripts can be freely downloaded from https://github.com/PrincetonUniversity/shae-cellshape-public.

1. Make a folder inside a folder on the desktop that contains the cropped images and the cell_shape_settings_tri.txt file from shaecellshape-public/exampleData_tri.

2. Edit cell_shape_setting_tri.txt to have the correct settings for the experiment of interest. For this experiment, the settings file includes the following lines:

nm_per_pixel 70

Z_scale 0.65

stack_z_size 41

stack_t_size 1

Fstack_z_size 41

Fstack_t_size 1

stack_seperation_nm 100

Fstack_seperation_nm 100

psfScript -999 osuPSF20180726

gradient 1

NOTE: This text file is organized into sections and each section is parsed into its own variable. While many of the settings do not need to be changed from experiment to experiment, one should make sure that the size of the z-stack (stack_z_size for shape, Fstack_z_size for protein of interest), spacing of the z-stack (stack_seperation_nm, Fstack_seperation_nm), relative focal shift of 
the microscope $\left(Z \_s c a l e\right)^{23}$, the pixel size in xy $\left(n m \_p e r \_p i x e l\right)$, and the name of the script that loads the blurring function of the microscope (psfScript) match the experiment. The gradient field should be set to 1 if the shape channel is cytoplasmically filled and 0 if it is a membrane-stained object.

3. Run Cell_shape_detector3dConvTriFolder function (shae-cellshape-public/CellShapeDetectorTri/) with the string to the folder location followed by the number of the cell to start on, and the number of cells you want to run.

NOTE: An example for the input will look as follows: Cell_shape_detector3dConvTriFolder ('path to folder with cropped images', starting index in folder, \# of cells). A typical cell may take between 5-20 min for the reconstruction to converge and finish.

4. Screen the cell reconstructions to ensure that they are correct before using the cells for any statistical analysis.

1. Run ScreenFits (shae-cellshape-public/shae-fitViewerGui/) to visually screen individual cell reconstructions.

2. Click the select folder button when the graphical user interface (GUI) opens, then select the folder with the reconstruction data files (TRI.mat) created in step 5.3.

3. Select the box next to the cell reconstruction if a cell appears misshapen or did not fully converge (Figure 3). This could look like a cell with a hole, a flat side, or a branch coming out of it. This will append 'FLAG' to the file name so that it can be excluded from any downstream analysis.

NOTE: The reconstructed cell can be compared to the original images. This can be especially important if your cells come from a various heterogenous population of shapes.

5. Run enrichmentSmoothingSpline (shae-cellshape-public/) to create an enrichment profile of the relative concentration of the protein of interest as a function of the Gaussian curvature at the surface.

1. Select each folder with TRI.mat files created in step 5.3.3.

2. Select the newly created (5.5.1) curve.mat file.

NOTE: A curve.mat file will be created for each folder that was selected in 5.5.1. All of the enrichment profiles will be presented on one graph. If individual graphs are required than run 5.5 for each folder.

NOTE: In addition to the precise geometric localization of a fluorescent protein, there are many other ways to analyze the data from the secondary channel, including counting the number, size, and orientation of objects ${ }^{4}$.

\section{Representative Results}

Bacteria come in a wide variety of shapes and sizes that may determine their functions in nature ${ }^{1}$. The outcome of this procedure is an accurate $3 \mathrm{D}$ representation of cells from the forward convolution of a z-stack of images (Figure 1). This method is especially important when dealing with curved cells (Figure 2), or with abnormally shaped cells (Figure 4A), as a 2D representation does not reflect the curvature of the cells accurately. In order to use the forward convolution method (Figure 1A), cells need to be either peripherally stained or have a cytoplasmic stain (Figure 2B left vs. right).

Figure 4 shows MreB localization in the cell. A GFP fusion was made to the bacterial actin protein MreB $^{21}$ in order to study its precise localization in both wild type and mutant $E$. coli cells ${ }^{4}$. Because MreB is associated with the membrane, 3D imaging is required to faithfully reproduce its position in the cell. By making these measurements in 3D, we were able to reconstruct the shapes of both wild type and rodZ mutant cells (Figure 4A). The localization of MreB was shown to be enriched at small Gaussian curvatures, a geometric feature that can only be measured in 3D, in a RodZ dependent manner (Figure 4B). 


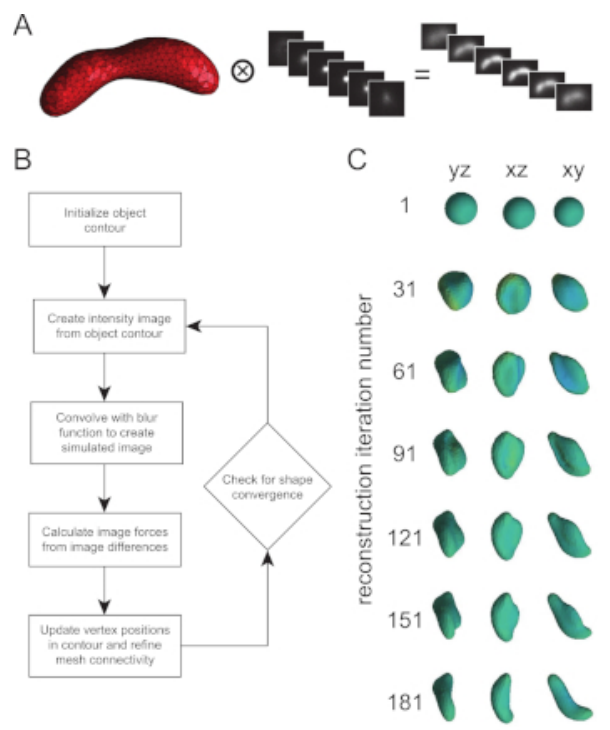

Figure 1: Forward convolution method reconstructs cells with no prior knowledge of cell shape. (A) The final output of the method is a 3D cell reconstruction derived by comparing a test shape with the calibrated blurring function of the microscope. This is compared with the observed z-stack until the observed 3D image matches the hypothetical one. The image shown here is a rendering of the reconstruction of one C. crescentus cell. (B) The reconstruction pipeline iteratively updates the estimated positions of the surface elements based on the observed image stack. For full algorithmic details of the method, see Nguyen ${ }^{3}$. (C) The reconstruction pipeline starts with no a priori knowledge of the shape of the cell and updates the position and number of surface vertices to match the observed z-stack. Representative images from every 30 steps during the 3D reconstruction are shown from three different angles of a $V$. cholerae cell. The surface positions of this shape are updated to minimize the difference between the simulated and observed stacks. Please click here to view a larger version of this figure.

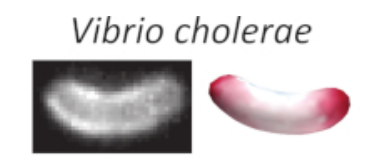

Helicobacter pylori
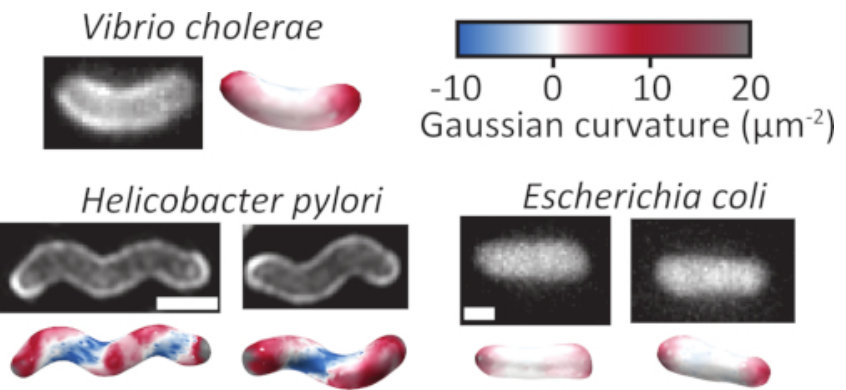

Figure 2: Representative 3D reconstructions of three different shaped bacterial species. Three-dimensional reconstructions can be made from cells with their membrane stained (left) or filled with a cytoplasmic fluorophore (right). The shape and curvature of the starting cell is not important, as a bent rod, twisted rod, or straight rod are all able to be accurately reconstructed. The reconstructed surfaces are colored based on the local Gaussian curvature of the surface. Scale bar $=1 \mu \mathrm{m}$. Please click here to view a larger version of this figure. 
pass quality control

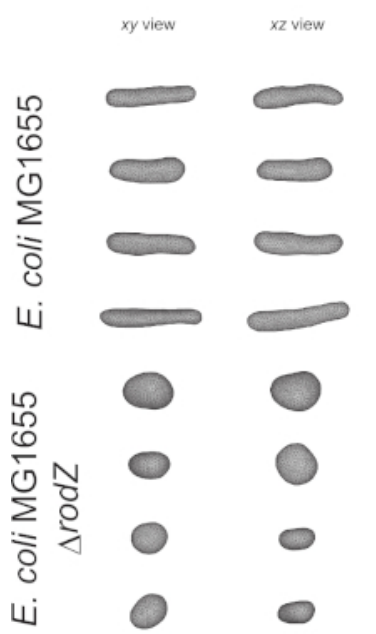

fail quality control

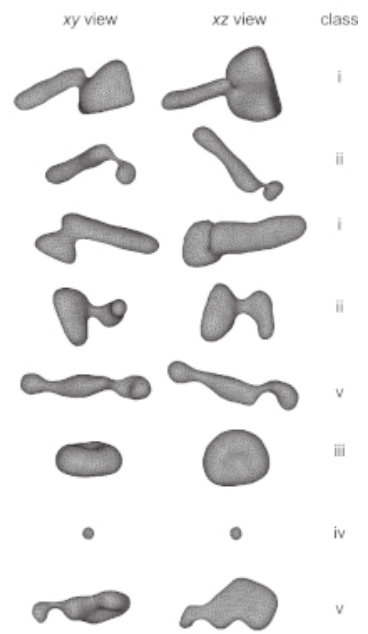

Figure 3: Reconstructions can fail for multiple reasons. Cells must be screened to ensure that the reconstruction algorithm converged to a reasonable result. Left $=$ representative wild type (top) and rodZ mutant (bottom) $E$. coli cells that have passed quality control. Right $=$ cells that failed to reconstruct properly and did not pass quality control. Five classes of failed convergence are shown: (i) cells that are too close to the edge of the cropping region leading to a sharp demarcation, (ii) cells that are too close to another cell, (iii) cells that produced a divot, (iv) cells that did not proceed past the initial starting point, and $(v)$ other unknown errors. Please click here to view a larger version of this figure.

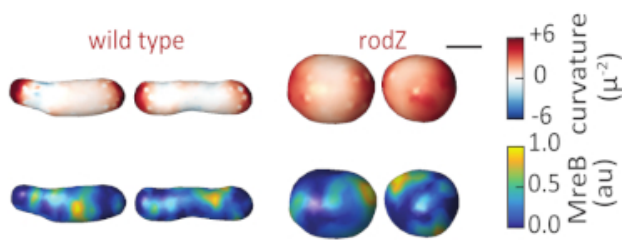

B

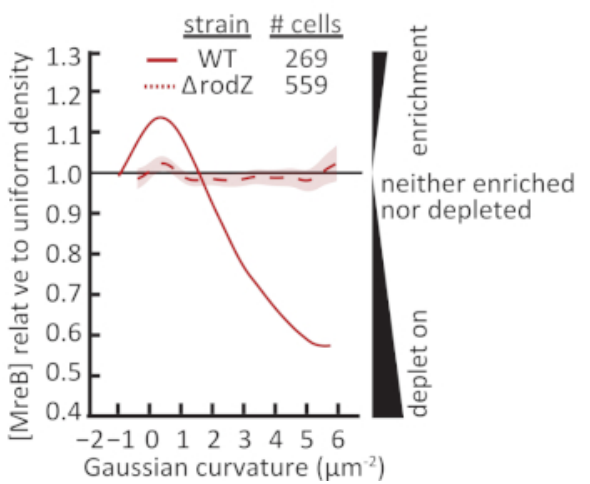

Figure 4: Representative data showing protein localization to specific cellular geometries. (A) Three-dimensional reconstructed cells of wild type and rodZ E. coli cells with Gaussian curvature and MreB fluorescence intensity displayed. Scale bar $=1 \mu \mathrm{m}$. (B) Enrichment plots of MreB from wild type and rodZ E. coli cells. Values $>1$ show enrichment and values $<1$ show depletion of MreB from these cellular regions relative to uniform coverage of the cell. Shaded areas indicate mean $\pm 90 \%$ bootstrap confidence interval of the mean. The curve for each strain is a cubic smoothing spline and is truncated using a probability threshold for extreme curvatures of $p>5 \times 10^{-3}$. This figure has been modified from (Bratton et al. $)^{4}$. Please click here to view a larger version of this figure.

\section{Discussion}

A critical step in this protocol is the acquisition of high-quality images. To properly reconstruct the cells, there must be enough blurring above and below the cell. Therefore, it is imperative that the z-stack taken covers a large enough distance. The number of steps taken during the image acquisition can be adjusted for each strain. For example, E. coli cells deleted for rodZ are wider and require more steps, and therefore, a greater distance, than wild type cells. If the sample drifts during image acquisition, the reconstruction can have major errors. Therefore, it is important to let the slide come to thermal equilibrium with the microscope before imaging to avoid drift during the z-stack acquisition. Cells should be imaged on pads with low autofluorescence. Media components, such as those found in the common LB medium, have autofluorescence that can cause problems when trying to reconstruct the cells. The density of cells on the imaging pad is important because the reconstruction process is performed independently on each cell. Too few cells will increase the time needed to obtain images of a sufficient number of cells, while too 
many cells will result in imaging fields that are too dense to easily crop individual cells. Because not all cells reconstruct properly, extra cells should be imaged during the acquisition step and all outputs should be screened before moving forward with statistical analysis (Figure 3 ).

Many of the limitations for this method are technical. On the microscope to be used, one must have an objective that has a high numerical aperture (typically $>1.4$ ), because this enables optical sectioning on the size scale of bacteria. Additionally, the microscope needs to be equipped with a piezo stage that can take small, precise steps in the z-direction. Furthermore, while it is not necessary, access to high-throughput computational resources to run the image analysis software is highly recommended because it will reduce the processing time to reconstruct cells.

One conceptual limitation to the method is that the correct energy scales for weighting the smoothness of the reconstruction relative to the signal-to-noise ratio of the images must be chosen. To validate a choice of parameters, the sizes and shapes of cells should be measured using independent methods such as transmission electron microscopy (TEM) or atomic force microscopy (AFM). As a proof of principle, 3D reconstructions of cells were performed either on an AFM to test for z-accuracy $(<50 \mathrm{~nm})$ or on a TEM grid to test for xy-accuracy $(<30 \mathrm{~nm})^{3}$ Such a correlated approach is time consuming and costly. A simpler approach may be to image standard samples such as wild type cells or $1 \mu \mathrm{m}$ spherical beads. The diameter and sphericity of the reconstructions can be used to ensure that the size and energy scales used in the reconstruction are correct.

This is not the only method that seeks to extract high resolution spatial information from fluorescence microscopy images. Many review articles describe recent advances in the field of super-resolution microscopy ${ }^{24,25}$. Resolution-enhancing techniques such as deconvolution microscopy ${ }^{26}$ spinning disk confocal microscopy ${ }^{27}$, pixel reassignment ${ }^{28}$, and structured illumination microscopy (SIM) ${ }^{29}$ seek to improve the resolution of the images acquired by the microscope. These methods are not incompatible with the approach presented. Recently this method was adapted to allow for SIM-based images as inputs ${ }^{9}$. While the forward convolution method shares some of its underpinnings with deconvolution microscopy, it has a completely different output. Whereas approaches such as deconvolution microscopy seek to improve the resolution of the image, this approach does not generate an image but rather a cell shape reconstruction with roughly $50 \mathrm{~nm}$ precision. Single-molecule active-control microscopy techniques based on sparsely labeled samples can provide even higher levels of spatial precision than this method. In many cases, these single molecule approaches require optimization of the fluorescent constructs and can require long acquisition times, making them difficult to use with live or dynamic samples. Each of these methods comes with one or more caveats that this method does not. For instance, the benefits advertised by spinning disk confocal microscopy are not as applicable to monolayers of bacterial cells, where there is not much out of plane light. Furthermore, this method provides a pipeline to acquire accurate 3D cell shapes and protein localization without the need of any specialized fluorophores. This method has minimal hardware requirements (i.e., z piezo, high NA objective) and requires only tens of images per timepoint, allowing one to easily investigate dynamic 3D structures ${ }^{6}$.

There have been an increasing number of approaches to study the organization of bacterial cells in 3D structures. These include approaches conceptually similar to this that take advantage of high quality, 3D fluorescence images ${ }^{30,31,32}$. This approach requires well isolated cells and makes no a priori assumptions about cellular geometry. However, to move into dense cellular aggregates or biofilms, the cells are assumed to be rod-like. This lower resolution view still enables investigating packing arrangements of the cells, although the high density of cells in the biofilm prevents analysis of the subcellular localization of specific factors.

In the future, it may be interesting to develop a framework to integrate the single molecule and wide-field approaches with this $3 \mathrm{D}$ reconstruction technique. Moreover, it may be possible to include this forward convolution approach with machine vision segmentation tools ${ }^{32}$ to allow for reconstructions of more dense cell clusters.

Why cells evolved into specific shapes is a complex issue that must reflect the complex environment in which they live. Understanding the evolution and function of cell shapes requires taking precise and accurate measurements of those shapes, which is what this method provides.

\section{Disclosures}

The authors have nothing to disclose.

\section{Acknowledgments}

We would like to thank Jeffrey Nguyen and Joshua Shaevitz for helping to develop this method.

Funding: RMM - NIH F32 GM103290-01A1, BPB - Glenn Centers for Aging Research and National Science Foundation PHY-1734030.

\section{References}

1. Young, K. D. The Selective Value of Bacterial Shape. Microbiology and Molecular Biology Reviews. 70 (3), $660-703$ (2006).

2. Persat, A., Stone, H. A., Gitai, Z. The Curved Shape of Caulobacter crescentus Enhances Surface Colonization in Flow. Nature Communications. 5, 3824 (2014).

3. Nguyen, J. P., Bratton, B. P., Shaevitz, J. W. in Bacterial Cell Wall Homeostasis: Methods and Protocols. (ed. Hee-Jeon Hong) Springer New York, 227-245 (2016).

4. Bratton, B. P., Shaevitz, J. W., Gitai, Z., Morgenstein, R. M. MreB Polymers and Curvature Localization are Enhanced by RodZ and Predict E. coli's Cylindrical Uniformity. Nature Communications. 9 (1), 2797 (2018).

5. Ouzounov, N. et al. MreB Orientation Correlates with Cell Diameter in Escherichia coli. Biophysical Journal. 111 (5), $1035-1043$ (2016)

6. Morgenstein, R. M. et al. RodZ links MreB to Cell Wall Synthesis to Mediate MreB rotation and Robust Morphogenesis. Proceedings of the National Academy of Sciences. 12510-12515 (2015).

7. Ursell, T. S. et al. Rod-Like Bacterial Shape is Maintained by Feedback Between Cell Curvature and Cytoskeletal Localization. Proceeding of the National Academy of Science U.S.A. 111 (11), E1025-E1034 (2014). 
8. Bartlett, T. M. et al. A Periplasmic Polymer Curves Vibrio cholerae and Promotes Pathogenesis. Cell. 168 (1-2), 172185.e115 (2017).

9. Taylor, J. A. et al. Distinct Cytoskeletal Proteins Define Zones of Enhanced Cell Wall Synthesis in Helicobacter pylori. bioRxiv. 545517 (2019).

10. Egan, A. J. F., Vollmer, W. The Physiology of Bacterial Cell Division. Annals of the New York Academy of Science. 1277 (1), 8-28 (2013).

11. Adams, D. W., Errington, J. Bacterial Cell Division: Assembly, Maintenance and Disassembly of the Z ring. Nature Reviews Microbiology. 7 (9), 642-653 (2009).

12. Werner, J. N., Gitai, Z., Melvin, I. S., Brian, R. C., Alexandrine, C. High-Throughput Screening of Bacterial Protein Localization. Methods in Enzymology. 471, 185-204 (2010).

13. de Pedro, M. A., Quintela, J. C., Höltje, J. V., Schwarz, H. Murein Segregation in Escherichia coli. Journal of Bacteriology. 179 (9), $2823-2834$ (1997).

14. Hussain, S. et al. MreB Filaments Align Along Greatest Principal Membrane Curvature to Orient Cell Wall Synthesis. eLife. 7, e32471 (2018).

15. Wong, F. et al. Mechanical Strain Sensing Implicated in Cell Shape Recovery in Escherichia coli. Nature Microbiology. 2, 17115 (2017).

16. Wong, F., Garner, E. C., Amir, A. Mechanics and Dynamics of Translocating MreB Filaments on Curved Membranes. eLife. 8, e40472 (2019).

17. Quint, D. A., Gopinathan, A., Grason, G. M. Shape Selection of Surface-Bound Helical Filaments: Biopolymers on Curved Membranes. Biophysical Journal. 111 (7), 1575-1585 (2016).

18. Colavin, A., Shi, H., Huang, K. C. RodZ Modulates Geometric Localization of the Bacterial Actin MreB to Regulate Cell Shape. Nature Communications. 9 (1), 1280 (2018).

19. Valap Sealant. Cold Spring Harbor Protocols. 2015, http://cshprotocols.cshlp.org/content/2015/2/pdb.rec082917 (2015).

20. Shannon, C. E. Communication in the Presence of Noise. Proceedings of the IRE. 37 (1), 10-21 (1949).

21. Billings, G. et al. De Novo Morphogenesis in L-Forms Via Geometric Control of Cell Growth. Mol Microbiol. 93 (5), $883-896$ (2014).

22. Sturrman, N. Measuring a Point Spread Function. iBiology. https://www.ibiology.org/talks/measuring-a-point-spread-function/ (2012).

23. Bratton, B. P., Shaevitz, J. W. Simple Experimental Methods for Determining the Apparent Focal Shift in a Microscope System. PLOS ONE. 10 (8), e0134616 (2015).

24. Moerner, W. E., Shechtman, Y., Wang, Q. Single-molecule Spectroscopy and Imaging over the decades. Faraday Discussions. 184, 9-36 (2015).

25. Sahl, S. J., Hell, S. W., Jakobs, S. Fluorescence Nanoscopy in Cell Biology. Nature Reviews Molecular Cell Biology. 18, 685 (2017).

26. Sarder, P., Nehorai, A. Deconvolution Methods for 3-D Fluorescence Microscopy Images. IEEE Signal Processing Magazine. 23 (3), $32-45$ (2006).

27. Oreopoulos, J., Berman, R., Browne, M. in Methods in Cell Biology. Vol. 123 (eds Jennifer C. Waters, Torsten Wittman) Academic Press, 153-175 (2014).

28. Huff, J. The Airyscan detector from ZEISS: confocal imaging with improved signal-to-noise ratio and super-resolution. Nature Methods. 12, 1205 (2015).

29. Gustafsson, M. G. L. Surpassing the Lateral Resolution Limit by a Factor of Two Using Structured Illumination Microscopy. Journal of Microscopy. 198 (2), 82-87 (2000).

30. Lee, M. K., Rai, P., Williams, J., Twieg, R. J., Moerner, W. E. Small-Molecule Labeling of Live Cell Surfaces for Three-Dimensional SuperResolution Microscopy. Journal of the American Chemical Society. 136 (40), 14003-14006 (2014).

31. Yan, J., Sharo, A. G., Stone, H. A., Wingreen, N. S., Bassler, B. L. Vibrio cholerae Biofilm Growth Program and Architecture Revealed by Single-Cell Live Imaging. Proceedings of the National Academy of Sciences. 113 (36), E5337-E5343 (2016).

32. Wang, J. et al. Bact-3D: A level set segmentation approach for dense multi-layered 3D bacterial biofilms. 2017 IEEE International Conference on Image Processing (ICIP). 330-334 (2017). 